\title{
Evaluation of Endophytes Isolated from \\ Rice Leaves for Their Antifungal \\ Activities Against Pyricularia oryzae \\ Causative Blast Disease \\ Mona Saleh M.
}

Rice Pathol. Res. Dept., Plant Pathol. Res. Inst., ARC, Egypt.

ice crop is attacked during its growing season by many fungal
diseases. Blast disease, caused by Pyricularia oryzae
(Magnaporthe grisea Hebert), is the major serious one. The aim of this
work was to replace fungicide applications by ecofriendly and natural
substances against the disease. Fifty endophyte isolates of different
microorganisms were isolated from rice leaves at Sakha Agricultural
Research Farm on five different isolation media. In vitro test was
performed to screen the antifungal activity of these isolates against rice
blast fungus. Of these, only five isolates showed highly antagonistic
activity in vitro and in vivo experiments. The secondary antifungal
metabolites of these endophyte isolates were extracted by TLC and
tested for their efficiency against the pathogen in in vitro test. Two
isolates were the most potent and identified as Streptomyces
albidoflavus strain Emeranaa MK203832.1 and Bacillus subtillus. GC-
MS extract analysis for these two isolates identified 24 and 13
compounds, respectively.

Keywords: Antimicrobial compounds, Endophytes, GC-MS analysis, Pyricularia oryzae, Rice blast and TLC test.

Application of fungicides, which leads to many human and animal diseases besides disorder in the bioenvironmental balance, has forced many investigators to explore a solution from this environment (Sukanya et al., 2011 and Zhang et al., 2016). To produce new bioactive molecules with higher efficiency, natural products i.e. plants and microbes are used. One of those important sources of natural bioactive compounds is endophytes. Endophytes are microorganisms which can colonize inner tissues of plants for part or all of their life time producing various enzymes, siderophores or antibiotics, which provide a protection to plants from phytopathogens or insects (Kafur \& Khan, 2011). Also, they may enhance their host growth by improving nutrient absorption. Rice plants are inhabited with various endophyte populations which enhance nitrogen fixation, promote plant growth and induce disease resistance (Tian et al., 2004; Fernandez et al., 2006 and Naik et al., 2009). Endophytic bacteria and streptomyces with antibacterial or antifungal activity were detected in many crops i.e. banana; common bean and rice (Tian, et al., 2004; Harish et al., 2008; Suryadi et al., 2011 and Costa et al., 2012).

Beneficial Bacillus spp. strains can compete other microbes that could affect crops; they can inhibit phytopathogenic attacks, or induce host-plant defense system against potential pathogenic attacks, stimulate plant growth, improve nutrient uptake and reduce some negative environmental traits (Brader et al., 2014 and Santoyo et al., 
2016). Bacillus species are known to have a wide spectrum of plant protection and growth promoting abilities. Species of Bacillus have the ability to produce endospores with a prolong viability and high resistance (Sella et al., 2014 and Noell et al., 2015) which makes them easy to be formulated in various types and stored in simple conditions, marketing them similar to chemical fungicides in a certain manner. Bacillus species could act directly against pathogens by producing extracellular lytic enzymes and secondary metabolites with inhibitory growth action (Kumar et al., 2012). They can also improve nutrient uptake or compete plant pathogens for the available nutrients (Borriss, 2011 and Chowdhury et al., 2013). In addition, reducing infection process by inducing defense responses in their host plants (Choudhary and Johri, 2009).

Streptomyces is the largest genus of Actinobacteria following Streptomycetaceae family (Hong et al., 2009). Over 500 species of Streptomyces genus have been characterized and about two third of the naturally antibiotics are produced by Streptomycetes (Mohanraj and Sekar, 2013).

Rice plants are subjected to attack by many fungal diseases during the season. Blast disease incited by Pyricularia oryzae Cavara is the most major and destructive one occurring all over rice growing areas of the world causing yield losses up to $50 \%$ (Ou, 1985). Therefore, the present work has been performed to isolate these endophytes, identify, evaluate their antagonistic ability in vitro and in vivo against rice blast causal fungus and finally to purify and detect its antifungal metabolites.

\section{Materials and Methods}

Sample collection:

About three healthy rice leaves adjacent to blast infected ones were collected from each of 15 individual plants of Sakha $101 \mathrm{cv}$. during 2017 rice growing season at Sakha Agricultural Research Station Farm. Samples were packed in clean paper bags, then directly transferred to the laboratory for isolation.

Isolation of endophytes:

To isolate the available number of endophytes, five different media; Water-Yeast Extract Agar (WYE), Yeast Extract-Malt Extract (ISP2), Zhang' Starch Soil Extract Agar (ZSSE agar), Bendict's modified from that of Lindenbein medium and Casein Starch medium (Crawford et el.,1993; Shriling and Gottlieb, 1966; Zhang, 2011; Porter et al., 1960 and Kuster \& Williams, 1964) were used. All isolation media were prepared in distilled water and adjusted with $0.1 \mathrm{~N} \mathrm{NaOH}$ to $\mathrm{pH} 7.1-7.2$ prior to autoclaving. Ten- gram samples of the collected healthy rice leaves were washed with running tap water for 10 minutes. Leaves were left till dryness, then cut into small pieces $(2 \mathrm{~cm})$ and surface sterilized by immersing in $0.5 \%$ sodium hypochlorite solution for 2 minutes (Okazaki, 2003). Sterilized pieces were directly rinsed with sterilized water three times. The samples were mashed in $90 \mathrm{ml}$ sterile distilled water with a mortar and pestle. A $0.1 \mathrm{ml}$ of this suspension was inoculated using the spread plate method. Three replicates of each of the aforementioned media were inoculated. Plates were incubated at $28^{\circ} \mathrm{C}$ for $15-30$ days. Pure cultures were maintained on PDA slants as stock cultures and then were stored at $4^{\circ} \mathrm{C}$ for further studies. 
Antifungal activity against Pyricularia oryzae in vitro:

The obtained microbial endophyte isolates were screened for their antifungal activity against Pyricularia oryzae using the dual culture technique (Gupta et al., 2001). All isolates were subjected to standardized test as follows: PDA medium was poured into $9 \mathrm{~cm}$ Petri dishes $(15 \mathrm{ml} /$ dish). After solidification, each plate was inoculated in the center with a disc $(6 \mathrm{~mm}$ in diameter) from 7- day old cultures of $P$. oryzae isolate. Two days later, plates were inoculated with a streak from each of the tested endophytic isolates (4 Isolate/plate) at the periphery. Three replicates were performed. Plates free from endophytic isolates were served as control. Plates were incubated at $28^{\circ} \mathrm{C}$ until full growth of control treatments. Inhibition zone of each antagonistic isolate was measured and the relative power of antibiosis (RPA) of each isolate was estimated through the ratio as described by Saleh, 2012 as follows:

$$
\mathrm{RPA}=\frac{z}{c}
$$

Where:

$\mathrm{Z}=$ Diameter of inhibition zone.

$\mathrm{C}=$ Diameter of spotted antagonistic isolate.

Identification of the potent bioagents:

Pure cultures of the two most efficient antagonistic endophyte isolates were identified. On the basis of $16 \mathrm{~S} \mathrm{r}$ RNA amplification, the first isolate (no.12) was identified at GATC company, Germany. A pair of universal primers $27 \mathrm{f}\left(5^{\prime}-\mathrm{CCG}\right.$ TCG ACG AGC TCA GAG TTT GAT CCT GGC TCA G- 3') and 1392r (5'-CCC GGG TAC CAA GCT TAA GGA GGT GAT CCA GCC GCA-3') were used. The sequence data were aligned and identified using the basic local alignment search tool (BLAST) in NCBI (https://blast.ncbi.nlm.nih.gov/Blast.cgi). MEGA X software was used to estimate the phylogenetic relations of the endophytic isolates. The evolutionary history was inferred using the Neighbor-Joining method (Saitou and Nei, 1987). The evolutionary distances were computed using the Maximum Composite Likelihood method (Tamura et al., 2004) and are in the units of the number of base substitutions per site. The analysis involved 11 isolate sequences. All ambiguous positions were removed for each sequence pair. There were a total of 1576 positions in the final dataset. Evolutionary analyses were conducted in MEGA X (Kumar et al., 2018). Whereas, the other isolate (no.17) was identified at Cairo Mircen, Faculty of Agriculture, Ain Shams University by GEN III Biolog system (Biolog, Inc., USA), Plaza et al., 2015.

Effect of the filtrates of the potent endophyte isolates on blast disease fungus:

Agar disk-diffusion method was followed for testing the antifungal activity of the two potent isolate filtrates (Bauer et al., 1966). The effect of culture filtrates of the two potent endophyte isolates on the radial growth of $P$. oryzae was studied following the method of Burgess et al. (1997). Flasks containing $100 \mathrm{ml}$ sterilized PD broth medium were inoculated with $0.5 \mathrm{~cm}$ diameter PDA mycelial disks of each isolate of isolates no.12 and 17. Each flask received one disk individually. The flasks were incubated at $30^{\circ} \mathrm{C}$ for 10 days for actinomyces isolate, while the bacterial isolate was incubated at $28^{\circ} \mathrm{C}$ for two days using shaking incubator $(100 \mathrm{rpm} / \mathrm{min})$. 
The cultural mats were removed and the rest of the medium was subjected to sterilization using $0.22 \mathrm{Mm}$ Millipore filter. The sterilized filtrate of each isolate was added individually to semi cold-autoclaved PDA medium with a concentration of 15 $\mathrm{ml} / 45 \mathrm{ml}$ medium, and, then poured into Petri plates. Sterilized water was added to check treatment. A $6 \mathrm{~mm}$ in diameter of PDA medium P. oryzae mycelium disks were placed in the center of each treated plate. Plates were incubated at $28^{\circ} \mathrm{C}$. Three replicates were prepared for each treatment. Experiment was noticed till full growth of control plates.

\section{Scanning Electron Microscope (SEM):}

To observe the significant effect of the most two potent antagonistic endophyte isolates (no.12 and 17) on the internal morphological changes of $P$. oryzae mycelium, Scaning Electron Microscope (SEM) was used. Samples were prepared according to Piroeva et al., (2013). The samples were then examined and photographed using a JEOL, JSM- 5200 LV scanning electron microscope, Japan at SEM Unit, Faculty of Science, Tanta University.

Antifungal activity against rice blast disease in vivo:

Susceptible rice cultivar Sakha 101 grains were sown in plastic trays. The trays were kept in the greenhouse at $25-30^{\circ} \mathrm{C}$. The seedlings were treated with the isolates, 3-4 weeks after sowing. The most five effective antifungal endophytes (no. 6, 12, 17,19 and 34) were tested for their efficiency in managing the blast disease infection on rice plants. The experiment was arranged in a randomized complete design with three replicates for each treatment. Two treatments were conducted comparing with beam fungicide. Firstly, we sprayed the filtrates of these endophyte isolates one day before inoculation with $P$. oryzae spores as a protective treatment. Secondly, the filtrates of the same five bioagent isolates were sprayed two days after inoculation with $P$. oryzae spores as a curative treatment. Beam fungicide was sprayed at $2 \mathrm{~g} / \mathrm{l}$. Spore suspension of $P$. oryzae was adjusted to $5 \times 10^{4}$ spores $/ \mathrm{ml}$ and sprayed using electrical spray gun. Trays with only the pathogen were left as a check treatment; likewise trays inoculated with only the bioagent were included to test for any probable side effects on plants. The inoculated trays were held in a dark moist chamber with $90-95 \%$ R.H. and $25-28^{\circ} \mathrm{C}$ overnight and then moved to the greenhouse conditions with saving sprinkling to enhance infection development. Blast reaction, as the typical blast lesions, according to the standard evaluation system using 0-9 scale (Anonymous, 1996) was recorded seven days after inoculation. Disease incidence and disease severity were recorded after full appearance of infection symptoms.

\section{Thin Layer Chromatography:}

The most five effective antifungal endophyte isolates (no. 6, 12, 17, 19 and 34) were grown on PD broth medium for 10 days at $30^{\circ} \mathrm{C}$, while, isolate no. 17 was grown for 48 hours $\left(150 \mathrm{rpm}, 28-30^{\circ} \mathrm{C}\right)$. Then, filtrates were centrifuged at 10,000 rpm for $10 \mathrm{~min}$. The culture media were extracted by mixing with ethyl acetate $1: 1$ $(\mathrm{v} / \mathrm{v})$ to separate the effective secondary metabolites of these five isolates using a thin layer chromatography (TLC) silica gel 60 F254 sheets (Merck, Darmstadt, Germany) (Wu et al., 2013). The resultant bands were viewed under UV light (wavelength $\lambda$ 254 and $\lambda-365 \mathrm{~nm}$ ) and bands boundaries were marked (Qin and Judith, 1999). Rf 
value was recorded. Then, discs of silica gel (where the separated secondary metabolites were adhered) were cut and tested for their antagonistic effect against 6 $\mathrm{mm}$ discs of $P$. oryzae from 7 days old cultures in Petri dishes using the dual culture technique in triplicate. Inhibition zone was visually recorded. Plates inoculated only with $P$. oryzae were left for comparison.

\section{Gas chromatography:}

The whole filtrates of the isolates that proved antifungal effect in both in vitro and in vivo experiments were analysed after extraction process with solvent on Agilant GC-MS system at The National Research Center, Egypt. Identification of different constituents was determined by comparing the spectrum fragmentation pattern with those stored in Wiley and NIST Mass Spectral Library data, (Kaaria et al., 2012).

Statistical analysis:

The randomized complete design was applied to both laboratory and greenhouse experiments. Means were compared using multiple range tests according to Duncn (1955).

\section{R e s u l t s}

Isolation of endophytes and antifungal activity against rice blast disease in vitro:

Isolation trials from healthy rice leaves grown on five different media resulted in the presence of fifty different endophytes isolates of both actinomyces and bacteria. These isolates were tested for their antifungal activity against Pyricularia oryzae in vitro (Table 1). Five isolates including four actinomycetes (no. 6, 12, 19 and 34) and one bacterial isolate (no.17) exhibited clear zones of fungal inhibition and highly RPA values. Of the five efficient antagonistic isolates, two isolates actinomyces no. 12 and bacteria no. 17 were the most potent as they recorded the highest RPA values, 10.88 and 12.83 , respectively.

\section{Identification of the potent bioagents:}

The isolates of the highest RPA ratio (no. 12 and17) were identified. Based on $16 \mathrm{~S}$ rRNA sequence analysis of potent bioagent isolates, actinomyces isolate no.12 was identified as Streptomyces albidoflavus 16S strain Emeranaa with accession no MK203832.1 in the Gene Bank. However, bacterial isolate no. 17 was identified by Biolog System as Bacillus subtillus. BLAST search analysis showed that the sequence was $94 \%$ similar to the $16 \mathrm{~S}$ sequence of both Streptomyces albidoflavus with accession no LN626361.1 and LN626360.1, respectively. While, it was 95\% similar to the $16 \mathrm{~S}$ sequence of Streptomyces albidoflavus isolate M-33 with accession no HG965213.1), (Fig.1). 
Table 1. Relative power of antibiosis (RPA) of fifty endophyte isolates against $P$. oryzae

\begin{tabular}{|c|c|c|c|}
\hline Isolate no. & RPA & Isolate no. & RPA \\
\hline 1 & $2.05^{\mathrm{jk} I}$ & 26 & $0.01^{\mathrm{q}}$ \\
\hline 2 & $2.5^{\mathrm{jk}}$ & 27 & $4.30^{\mathrm{fg}}$ \\
\hline 3 & $1.31^{\mathrm{mno}}$ & 28 & $4.60^{f}$ \\
\hline 4 & $2.47^{\mathrm{jk}}$ & 29 & $1^{\text {nop }}$ \\
\hline 5 & $0.66^{\mathrm{opq}}$ & 30 & $0.01^{\mathrm{q}}$ \\
\hline 6 & $9.64^{\mathrm{c}}$ & 31 & $0.01^{\mathrm{q}}$ \\
\hline 7 & $1.33^{\mathrm{mno}}$ & 32 & $0.01^{\mathrm{q}}$ \\
\hline 8 & $1.02^{\text {nop }}$ & 33 & $0.01^{\mathrm{q}}$ \\
\hline 9 & $0.44^{\mathrm{pq}}$ & 34 & $5.30^{\mathrm{de}}$ \\
\hline 10 & $0.88^{\text {nop }}$ & 35 & $0.01^{\mathrm{q}}$ \\
\hline 11 & $0.01^{\mathrm{q}}$ & 36 & $0.01^{\mathrm{q}}$ \\
\hline 12 & $10.88^{b}$ & 37 & $0.01^{\mathrm{q}}$ \\
\hline 13 & $0.01^{\mathrm{q}}$ & 38 & $1.83^{\mathrm{km}}$ \\
\hline 14 & $0.01^{\mathrm{q}}$ & 39 & $2.19^{\mathrm{jk}}$ \\
\hline 15 & $0.01^{\mathrm{q}}$ & 40 & $4.75^{\text {ef }}$ \\
\hline 16 & $0.01^{\mathrm{q}}$ & 41 & $2.55^{\mathrm{jk}}$ \\
\hline 17 & $12.83^{a}$ & 42 & $2.2^{\mathrm{jk}}$ \\
\hline 18 & $1.50^{\mathrm{Imn}}$ & 43 & $2.56^{\mathrm{jk}}$ \\
\hline 19 & $5.47^{\mathrm{d}}$ & 44 & $0.01^{\mathrm{q}}$ \\
\hline 20 & $1.26^{\mathrm{mno}}$ & 45 & $1.26^{\mathrm{mno}}$ \\
\hline 21 & $1.21^{\mathrm{mno}}$ & 46 & $3.62^{h}$ \\
\hline 22 & $1.08^{\text {nop }}$ & 47 & $2.25^{\mathrm{jk}}$ \\
\hline 23 & $1.16^{\mathrm{mno}}$ & 48 & $3.87^{\mathrm{gh}}$ \\
\hline 24 & $1.03^{\text {nop }}$ & 49 & $2.64^{i j}$ \\
\hline 25 & $3.25^{\mathrm{hi}}$ & 50 & $2.73^{i j}$ \\
\hline
\end{tabular}

In a column, means followed by a common letter are not significantly different at the $5 \%$ level by DMRT.

Effect of filtrates of the potent endophyte isolates on blast disease fungus:

The effect of Streptomyces albidoflavus 16S strain Emeranaa MK203832 and Bacillus subtillus filtrates on mycelial growth of P. oryzae is illustrated in Fig. 2. There was no growth in treated dishes for both isolates in comparison with the control treatment. However, Streptomyces albidoflavus 16S strain Emeranaa apparently exceeded the bacterial isolate which allowed growth of mycelium only on the disk. 


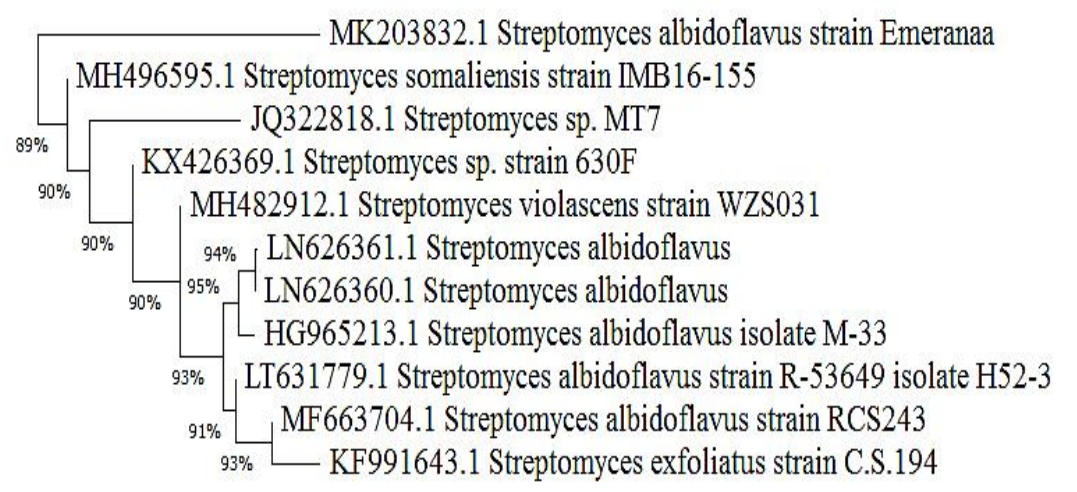

0.0020

Fig.1. Phylogenetic neighbour-joining tree showing the relationship of strain K203832 with elven members of the genus Streptomyces.

Effect of filtrates of the potent endophyte isolates on blast disease fungus:

The effect of Streptomyces albidoflavus 16S strain Emeranaa MK203832 and Bacillus subtillus filtrates on mycelial growth of P. oryzae is illustrated in Fig.(2). There was no growth in treated dishes for both isolates in comparison with the control treatment. However, Streptomyces albidoflavus 16S strain Emeranaa apparently exceeded the bacterial isolate which allowed growth of mycelium only on the disk.

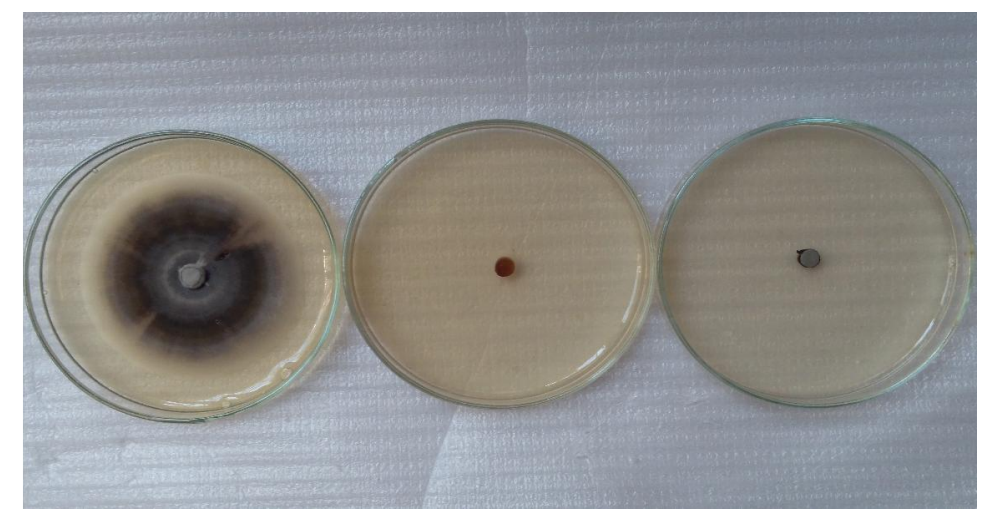

Fig. 2. Effect of culture filtrates of Streptomyces albidoflavus strain Emeranaa MK203832 (middle) and Bacillus subtillus (right) on growth of $P$. oryzae comparing with control treatment (left). 
Scanning Electron Microscope:

Using scanning electron macrograph, samples free from bioagents showed normal hyphae growth (Fig. 3a). Contrariwise, P. oryzae hyphae, was distorted when inoculated with the bioagents. Scanning confirmed the aggregation of the fungal mycelium. Hyphae were coiled, thickened (Fig.3: $12 \mathrm{~b} \& 17 \mathrm{~d}$ ) and cleaved (Fig.3: 12c \&17e) in the presence of Streptomyces albidoflavus 16S strain Emeranaa MK203832 and Bacillus subtillus, respectively.

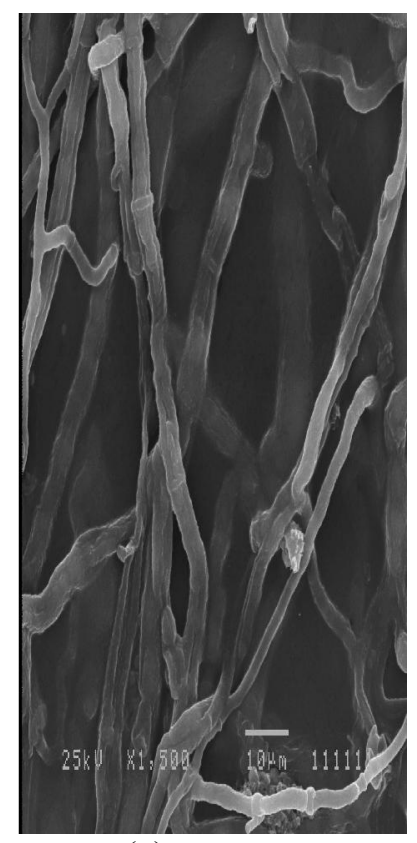

(a)

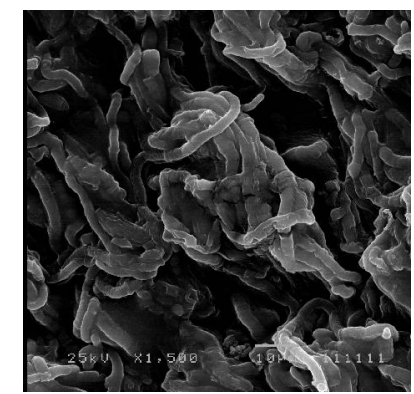

$12 \mathrm{~b}$

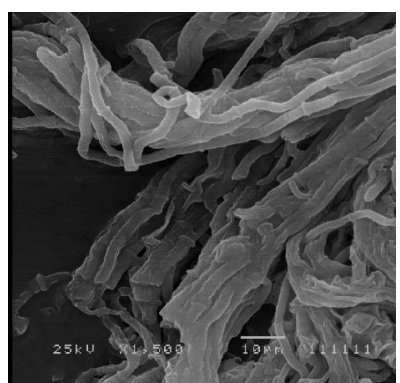

$17 \mathrm{~d}$

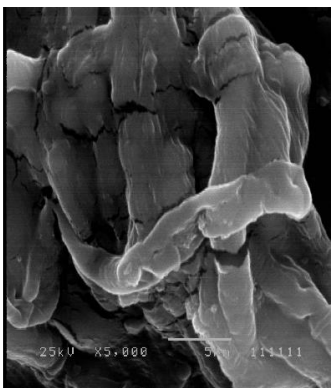

$12 \mathrm{c}$

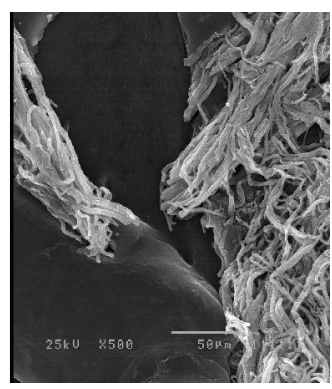

$17 \mathrm{e}$

Fig. 3. Scanning electron microscope photographs indicating the effect of Streptomyces albidoflavus 16S strain Emeranaa MK203832 (no. 12) and Bacillus subtillus (no.17) on P. oryzae mycelium: (a) control; 12b \& 17d, hyphae aggregation, coiling and thickening. $12 \mathrm{c} \& 17 \mathrm{e}$, hyphae cleavage.

Antifungal activity against rice blast disease in vivo:

Data presented in Table 2 show that, all the five tested endophytes were efficient against the rice blast disease in both treatments (spraying before or after inoculation with the pathogen), particularly, spraying after inoculation with the pathogen in comparison with the check treatment. However, Streptomyces albidoflavus 16S strain Emeranaa MK203832 isolate no.12 and Bacillus subtillus isolate no.17 proved to be the most efficient in managing the disease. They significantly recorded the least percentage of infection and severity after the fungicide treatment, alike they were applied before or after inoculation with the pathogen comparing with the other tested bioagents. Data also showed that treating plants with these isolates 
after pathogen inoculation was more efficient in controlling the disease than before pathogen inoculation. No side effects appeared on plants treated only with bioagents.

Table 2. Effect of antagonistic endophytes on rice blast disease incidence under greenhouse conditions

\begin{tabular}{|l|c|c|c|c|}
\hline \multirow{2}{*}{ Bioagent no. } & \multicolumn{2}{c|}{$\begin{array}{c}\text { One day Before } \\
\text { inoculation }\end{array}$} & \multicolumn{2}{c|}{$\begin{array}{c}\text { Two days After } \\
\text { inoculation }\end{array}$} \\
\cline { 2 - 5 } & \%Infection & Severity & \% Infection & Severity \\
\hline 1- Actinomyces no. 6 & $27.01 \mathrm{~d}$ & $1.34 \mathrm{~b}$ & $9.91 \mathrm{~b}$ & $0.79 \mathrm{~b}$ \\
\hline $\begin{array}{l}\text { 2-Streptomyces albidoflavus } \\
\text { strain Emeranaa MK203832 } \\
\text { (no.12) }\end{array}$ & $24.85 \mathrm{~d}$ & $1.25 \mathrm{~b}$ & $0.001 \mathrm{~d}$ & $0.001 \mathrm{e}$ \\
\hline 3- Bacillus subtillus (no.17) & $13.27 \mathrm{e}$ & $1.32 \mathrm{~b}$ & $0.001 \mathrm{~d}$ & $0.001 \mathrm{e}$ \\
\hline 4- Actinomyces no. 19 & $48.26 \mathrm{~b}$ & $1.46 \mathrm{~b}$ & $6.73 \mathrm{c}$ & $0.67 \mathrm{c}$ \\
\hline 5- Actinomyces no. 34 & $38.96 \mathrm{c}$ & $1.15 \mathrm{~b}$ & $0.93 \mathrm{~d}$ & $0.22 \mathrm{~d}$ \\
\hline 6- control & $75.00 \mathrm{a}$ & $3.50 \mathrm{a}$ & $76.00 \mathrm{a}$ & $1.96 \mathrm{a}$ \\
\hline 7- Beam (fungicide) & $0.001 \mathrm{f}$ & $0.001 \mathrm{c}$ & $0.001 \mathrm{~d}$ & $0.001 \mathrm{e}$ \\
\hline
\end{tabular}

In a column, means followed by a common letter are not significantly different at the $5 \%$ level by DMRT.

Thin Layer Chromatography:

The ethyl acetate extraction method was the best in extracting and separating the crude antimicrobial compounds from filtrates of the five antifungal endophytes isolates (act. no. 6, 12, 17, 19 and 34) by TLC. One or two bands were observed. Bioassay of TLC extract proved that the compounds showing antifungal activity migrated through the plate with $\mathrm{Rf}$ value ranging from 0.27 to 1.00 for all isolates. The compounds activity was confirmed by agar diffusion method for P. oryzae, Table 3 and Fig. (4).

Table 3. The five endophytes Rf value of formative peaks by TLC and their inhibition zone against the rice blast fungus

\begin{tabular}{|c|c|c|}
\hline Isolate peak, assigned substance & R.f. value & Inhibition zone \\
\hline Actino. 6 & 0.27 & + \\
\hline Actino 6 & 0.93 & + \\
\hline Actino 12 & 0.29 & ++ \\
\hline Actino 12 & 0.96 & ++ \\
\hline Bacteria 17 & 1 & ++ \\
\hline Actino 19 & 0.89 & + \\
\hline Actino 19 & 0.78 & + \\
\hline Actino 34 & 0.90 & + \\
\hline Actino 34 & 0.92 & + \\
\hline
\end{tabular}




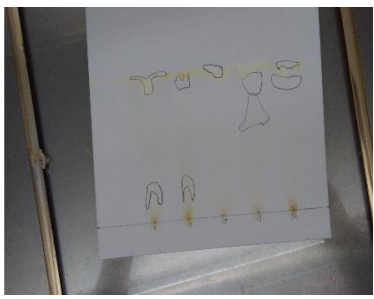

Isol.no.:6- 12- 17- 19-

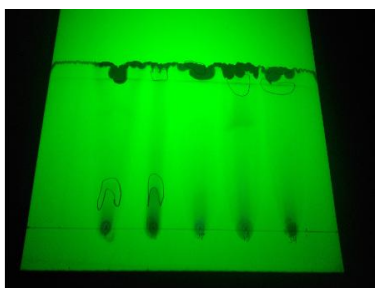

Isol.no.:6- 12- 17- 19-34

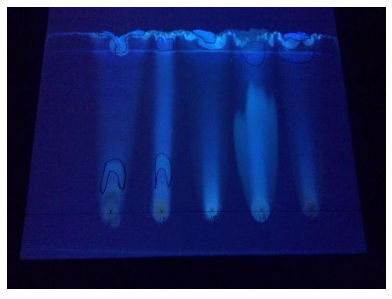

Isol.no.:6- 12- 17- 19-34

Fig. 4. Chromatogram of separated antifungal compounds by TLC

Identification of bioactive metabolites GC:

The identification of the compounds excreted by the most active biocontrol endophytes was confirmed based on the molecular formula and peak area \%. This area clarifies the quantity of the compound present in the active band. GC- MS analysis proved the presence of different compounds for both isolates which introduced 24 and 13 different compounds for Streptomyces albidoflavus strain Emeranaa MK203832.1 and Bacillus subtillus (Tables $4 \& 5$, respectively). These compounds enclosed phenols, antioxidants, fatty acids and antifungal compounds.

Table 4. Antimicrobial compounds identified from Streptomyces albidoflavus strain Emeranaa MK203832.1 through GC-MS analysis

\begin{tabular}{|c|l|c|c|c|}
\hline Peak & \multicolumn{1}{|c|}{ Compound name } & $\begin{array}{c}\text { Molecular } \\
\text { formula }\end{array}$ & $\begin{array}{c}\text { \%o } \\
\text { Peak } \\
\text { area }\end{array}$ & Property \\
\hline 1 & $\begin{array}{l}\text { Benzaldehyde,3-methoxy-4- } \\
\text { (trimethylsilyl)oxy]-, O- } \\
\text { methyloxime Or Vanillin, MO TMS }\end{array}$ & $\mathrm{C}_{12} \mathrm{H}_{19} \mathrm{NO}_{3} \mathrm{Si}$ & 2.03 & $\begin{array}{c}\text { Phenolic aldehyde, } \\
\text { antifungal, Carpinella } \text { et } \\
\text { al., 2003 }\end{array}$ \\
\hline 2 & Undecane & $\mathrm{C}_{11} \mathrm{H}_{24}$ & 13.80 & $\begin{array}{c}\text { Antifungal, Manna and } \\
\text { Kim, 2018 }\end{array}$ \\
\hline 3 & $\begin{array}{l}\text { 2-Pyridinepropanoic acid, alpha.- } \\
\text { methyl-.beta--oxo-, ethyl ester or } \mathrm{N}- \\
\text { acetyl-L-phenylalanine }\end{array}$ & $\mathrm{C}_{11} \mathrm{H}_{13} \mathrm{NO}_{3}$ & 0.72 & $\begin{array}{c}\text { Antimicrobial } \\
\text { antioxidant }\end{array}$ \\
\hline 4 & $\begin{array}{l}\text { 1,7-Di(2,5-dimethylphenyl)- } \\
2,2,4,4,6,6-h \text {-xamethyl-1,3,5,7- } \\
\text { tetraoxa-2,4,6-trisila }\end{array}$ & $\mathrm{C}_{22} \mathrm{H}_{36} \mathrm{O}_{4} \mathrm{Si}_{3}$ & 1.14 & N.I. \\
\hline 5 & $\begin{array}{l}\text { Cyclopentasiloxane, decamethyl- } \\
\mathrm{C}_{10} \mathrm{H}_{30} \mathrm{O}_{5} \mathrm{Si}_{5}\end{array}$ & 0.88 & $\begin{array}{c}\text { Precursor in industry } \\
\text { and medical field. CSR, } \\
\text { 2010 }\end{array}$ \\
\hline 6 & $\begin{array}{l}1,1,3,3,5,5,7,7-O c t a m e t h y l-7-(2- \\
\text { methylpropoxy)tetrasiloxan-1-ol }\end{array}$ & $\mathrm{C}_{12} \mathrm{H}_{34} \mathrm{O}_{5} \mathrm{Si}_{4}$ & 1.33 & N.I. \\
\hline 7 & $\begin{array}{l}1,1,1,3,5,7,9,9,9- \\
\text { Nonamethylpentasiloxane }\end{array}$ & $\mathrm{C}_{9} \mathrm{H}_{30} \mathrm{O}_{4} \mathrm{Si}_{5}$ & 0.86 & N.I. \\
\hline
\end{tabular}




\begin{tabular}{|c|c|c|c|c|}
\hline & & & & Continued \\
\hline 8 & 2,4-Di-tert-butylphenol & $\mathrm{C}_{14} \mathrm{H}_{22} \mathrm{O}$ & 4.14 & $\begin{array}{l}\text { Antifungal, antioxidant, } \\
\text { biosurfactant, } \\
\text { allelochemical, Varsha } \\
\text { et al., } 2015\end{array}$ \\
\hline 9 & Cyclohexasiloxane, dodecamethyl- & $\mathrm{C}_{12} \mathrm{H}_{36} \mathrm{O}_{6} \mathrm{Si}_{6}$ & 0.89 & $\begin{array}{c}\text { Antifungal } \\
\text { Moustafa et al., } 2013\end{array}$ \\
\hline 10 & Cyclooctasiloxane, hexadecamethyl- & $\mathrm{C}_{16} \mathrm{H}_{48} \mathrm{O}_{8} \mathrm{Si}_{8}$ & 0.68 & $\begin{array}{c}\text { Antibacterial, Hassan, } \\
2016 \\
\end{array}$ \\
\hline 11 & $\begin{array}{l}\text { Hexasiloxane, } 1,1,3,3,5,5,7,7,9,9,11,1 \\
\text { 1-dodecamethyl- }\end{array}$ & $\mathrm{C}_{12} \mathrm{H}_{38} \mathrm{O}_{5} \mathrm{Si}_{6}$ & 3.75 & $\begin{array}{l}\text { Antifungal, Sivanandhan } \\
\text { et al., } 2018\end{array}$ \\
\hline 12 & $\begin{array}{l}\text { Tetradecanoic acid, 12-methyl-, } \\
\text { methyl ester, (S)- }\end{array}$ & $\mathrm{C}_{16} \mathrm{H}_{32} \mathrm{O}_{2}$ & 1.16 & $\begin{array}{l}\text { Antifungal, antioxidant, } \\
\text { Liu et al. (2008) }\end{array}$ \\
\hline 13 & $\begin{array}{l}\text { 2-Pyridinepropanoic acid, .alpha.- } \\
\text { methyl-.beta.-oxo-, ethyl ester or N- } \\
\text { acetyl-L-phenylalanine }\end{array}$ & $\mathrm{C}_{11} \mathrm{H}_{13} \mathrm{NO}_{3}$ & 1.25 & $\begin{array}{l}\text { Antimicrobial } \\
\text { antioxidant }\end{array}$ \\
\hline 14 & $\begin{array}{l}\text { Hexadecanoic acid, methyl ester or } \\
\text { palmitic acid }\end{array}$ & $\mathrm{C}_{17} \mathrm{H}_{34} \mathrm{O}_{2}$ & 11.67 & $\begin{array}{c}\text { Antioxidant, } \\
\text { Antimicrobial, } \\
\text { pesticidal, nematicidal } \\
\text { Banaras } \text { et al., } 2017 \\
\end{array}$ \\
\hline 15 & Silicone grease, siliconfett & & 0.59 & N.I. \\
\hline 16 & $\begin{array}{l}\text { Cyclohexane, } 1,1 \text { '-[1-(2,2- } \\
\text { dimethylbutyl)-1,3- propanediyl] bis- }\end{array}$ & $\mathrm{C}_{21} \mathrm{H}_{40}$ & 0.76 & N.I. \\
\hline 17 & $\begin{array}{l}\text { 2-Pyridinepropanoic acid, .alpha.- } \\
\text { methyl-.beta.-oxo-, ethyl ester or N- } \\
\text { acetyl-L-phenylalanine }\end{array}$ & $\mathrm{C}_{11} \mathrm{H}_{13} \mathrm{NO}_{3}$ & 0.61 & $\begin{array}{c}\text { Antimicrobial } \\
\text { antioxidant }\end{array}$ \\
\hline 18 & $\begin{array}{l}\text { Hexadecanoic acid, 14-methyl-, } \\
\text { methyl ester or methyl palmitate }\end{array}$ & $\mathrm{C}_{18} \mathrm{H}_{36} \mathrm{O}_{2}$ & 0.81 & $\begin{array}{c}\text { Antimicrobial, Banaras et } \\
\text { al., } 2017\end{array}$ \\
\hline 19 & $\begin{array}{l}\text { 9-Octadecenoic acid (Z)-, } \\
\text { phenylmethyl ester or Oleic acid }\end{array}$ & $\mathrm{C}_{25} \mathrm{H}_{40} \mathrm{O}_{2}$ & 0.76 & $\begin{array}{l}\text { Antifungal, Liu et al., } \\
2008\end{array}$ \\
\hline 20 & $\begin{array}{l}\text { 9,12,15-Octadecatrienoic acid, } 2- \\
{[(\text { trimethylsilyl)oxy }]-1-} \\
{[[(\text { trimethylsilyl)oxy }] \mathrm{m} \text { or }} \\
\text { Linolinic acid }\end{array}$ & $\mathrm{C}_{27} \mathrm{H}_{52} \mathrm{O}_{4} \mathrm{Si}_{2}$ & 0.62 & $\begin{array}{l}\text { Antifungal-Antioxidant, } \\
\text { Liu et al., } 2008 \text { and Pohl } \\
\text { et al., } 2011\end{array}$ \\
\hline 21 & $\begin{array}{l}\text { 9-Octadecenoic acid (Z)-, methyl } \\
\text { ester }\end{array}$ & $\mathrm{C}_{18} \mathrm{H}_{34} \mathrm{O}_{2}$ & 4.80 & $\begin{array}{c}\text { Antioxidant, } \\
\text { Antimicrobial Abubakar } \\
\text { \& Majinda, (2016) }\end{array}$ \\
\hline 22 & $\begin{array}{l}\text { trans-9-Octadecenoic acid, pentyl } \\
\text { ester }\end{array}$ & $\mathrm{C}_{23} \mathrm{H}_{44} \mathrm{O}_{2}$ & 0.85 & Amyl elaidate \\
\hline 23 & Methyl stearate or Stearic acid & $\mathrm{C}_{19} \mathrm{H}_{38} \mathrm{O}_{2}$ & 5.36 & Antifungal- antioxidant \\
\hline 24 & Decyl oleate or Oleic acid & $\mathrm{C}_{28} \mathrm{H}_{54} \mathrm{O}_{2}$ & 40.54 & $\begin{array}{l}\text { Antifungal, Liu et } \\
\text { al.,2008 }\end{array}$ \\
\hline
\end{tabular}

N.I. $=$ Non identified 
Table 5. Antimicrobial compounds identified from Bacillus subtillus through GC-MS analysis.

\begin{tabular}{|c|c|c|c|c|}
\hline Peak & Compound Name & $\begin{array}{l}\text { Molecular } \\
\text { Formula }\end{array}$ & $\begin{array}{c}\text { Area } \\
\%\end{array}$ & Property \\
\hline 1 & Butyl alcohol 1-D1 & $\mathrm{C}_{4} \mathrm{H}_{10} \mathrm{O}$ & 44.57 & Biosurfactant \\
\hline 2 & Glycolaldehyde dimer & $\mathrm{C}_{4} \mathrm{H}_{8} \mathrm{O}_{4}$ & 5.25 & $\begin{array}{l}\text { Morpholin synthesis, } \\
\text { Kim et al., } 2001\end{array}$ \\
\hline 3 & Butanone,3-hydroxy- & & 9.4 & Biosurfactant \\
\hline 4 & 1,2,3-Propanetriol or glycerol & $\mathrm{C}_{3} \mathrm{H}_{8} \mathrm{O}_{3}$ & 1.51 & Glycerin \\
\hline 5 & $\begin{array}{l}\text { Acetic acid, hydroxy- or } \\
\text { Glycolic acid }\end{array}$ & $\mathrm{C}_{2} \mathrm{H}_{4} \mathrm{O}_{3}$ & 2.06 & Industrial uses \\
\hline 6 & 2,3-Butanediol, $\left[\mathrm{R}-\left(\mathrm{R}^{*}, \mathrm{R}^{*}\right)\right]-$ & $\mathrm{C}_{4} \mathrm{H}_{10} \mathrm{O}_{2}$ & 29.75 & Biosurfactant \\
\hline 7 & Undecane & $\mathrm{CH}_{3}\left(\mathrm{CH}_{2}\right)_{9} \mathrm{CH}_{3}$ & 0.65 & $\begin{array}{l}\text { Antifungal, Mannaa and } \\
\text { Kim, } 2018\end{array}$ \\
\hline 8 & 2,4-Di-tert-butylphenol & $\mathrm{C}_{14} \mathrm{H}_{22} \mathrm{O}$ & 0.65 & \begin{tabular}{|c|} 
Antifungal, antioxidant \\
Biosurfactant, Dharni et al. \\
2014
\end{tabular} \\
\hline 9 & $\begin{array}{l}\text { Hexadecanoic acid, methyl } \\
\text { ester or palmitic acid }\end{array}$ & $\mathrm{C}_{17} \mathrm{H}_{34} \mathrm{O}_{2}$ & 0.98 & $\begin{array}{c}\text { Antifungal, Liu et al.,2008 } \\
\text { Pohl, et al., 2011 }\end{array}$ \\
\hline 10 & $\begin{array}{l}\text { Octadecanoic acid, methyl ester } \\
\text { or Oleic acid }\end{array}$ & $\mathrm{C}_{27} \mathrm{H}_{56} \mathrm{O}_{2}$ & 0.48 & Antifungal, Liu et al., 2008 \\
\hline 11 & Butyl citrate & $\mathrm{C}_{18} \mathrm{H}_{32} \mathrm{O}_{7}$ & 0.79 & $\begin{array}{l}\text { Biosurfactant,plasticizer, } \\
\text { Takeshita } \text { et al., } 2011\end{array}$ \\
\hline 12 & $\begin{array}{l}\text { Hexanedioic acid, bis(2- } \\
\text { ethylhexyl) ester or DEHA }\end{array}$ & $\mathrm{C}_{22} \mathrm{H}_{42} \mathrm{O}_{4}$ & 0.74 & $\begin{array}{l}\text { Plasticizer, Bizzari et al. } \\
\text { 2009, cosmetic products, } \\
\text { Gottschalck and McEwen } \\
\text { 2004, and pesticides } \\
\text { PMRA 2005 }\end{array}$ \\
\hline 13 & Decyl oleate & $\mathrm{C}_{28} \mathrm{H}_{54} \mathrm{O}_{2}$ & 3.17 & $\begin{array}{l}\text { Antifungal and cosmetics } \\
\text { Liu et al., } 2008\end{array}$ \\
\hline
\end{tabular}

\section{Dis cus s i o n}

The obtained results showed that fifty endophytes were isolated from rice leaves on different five media. Only five isolates were effective against Pyricularia oryzae by inhibiting its growth on the tested media. Of these five isolates, two isolates were the most potent against $P$. oryzae and identified by $16 \mathrm{~S}$ rRNA sequence as Streptomyces albidoflavus Emeranaa with accession no. MK203832.1 (isolate no.12) and was confirmed to the species level. Similar findings were obtained by Cook and Meyers (2003). However, Bacillus subtillus (isolate no.17) was identified according to biolog system as adopted by Wang et al. (2012).

Concerning the effect of filtrates of these two isolates, the obtained results showed that they were effective in inhibiting the mycelial growth of $P$. oryzae on individually treated PDA plates with superiority of $S$. albidoflavus Emeranaa strain MK203832.1 in P. oryzae growth obstruction. This may be due to their ability to 
produce various metabolites that degrade the fungal cell walls, consequently inhibited the fungal growth of the pathogen. However, Alina et al. (2015) reviewed the ability of B. subtillus to produce different antifungal compounds i.e., chitinases, cellulolytic enzymes and antibiotic compounds. This was assured by scaning electron microscope technique which illustrated the distortion of P. oryzae hyphae. Thus, the production of antifungal metabolites by these endophytes makes them very promising biocontrol agents (Rajer et al., 2017).

The efficiency of spraying the filtrates of the five active endophytes in reducing blast disease incidence in both treatments was promising. Particularly, the filtrates of S. albidoflavus Emeranaa strain MK203832.1 and B. subtillus when applied two days after inoculation with the pathogen. This may be due to the ability of these endophytes to produce plethora of bioactive secondary metabolites. These metabolites hinder the pathogen growth, so no infection symptoms appear. Similar results were found on several crops by many investigators (Bressan, 2003; Taechowisan et al., 2003; Taechowisan, et al., 2005 and Gangwar et al., 2011) and this was assured by scanning electron microscope study.

S. albidoflavus Emeranaa strain MK203832.1 and B. subtillus recorded the highest $\mathrm{Rf}$ values in Thin layer chromatography test, which proved their most efficiency. So, this was promoting to more studies using GC-MS analysis to detect the bioactive compounds from the whole filtrates of these two isolates.

The detected compounds were categorized into phenols, antioxidants, alcohols and fatty acids. S. albidoflavus Emeranaa strain MK203832.1 and B. subtilis filtrates are known to produce many volatile organic compounds efficient in plant protection against pathogens whether as antioxidants or as antimicrobials (Agoramoorthy et al., 2007; chandrasekharan et al., 2008 and Lima et al., 2011). These toxic volatile compounds; hexadecanoic acid, 9- octadecenoic acid and 2,4-Di-tert-butylphenol; were detected also in some plants; Chenopodium album (root extract), grassy weeds or other microorganisms extracts, i.e. Lactococcus sp.; Pseudomonas monteilli and actinomycetes (Ali et al., 2017; Varsha et al., 2015). In the present study, the two bioagents contain some similar compounds (i.e., palmitic acid, oleic acid, 2,4-Di-tertbutylphenol, undecane and decyl oleate) which supposed them to be a promising source of antifungal compounds and industry for better human life (i.e., vanillin, glycolic acid and glycoaldehyde dimmer).

These findings are in accordance with many workers (Kadoma et al., 2009; Sang et al., 2011; Sang and Kim, 2012; Dharni et al., 2014 and Varsha et al., 2015) who reported that, 2,4-Di-tert-butylphenol (DTBP) is an antioxidant or antifungal biosurfactant compound. As it inhibits sporulation and hyphal growth of many pathogenic fungi. Bacillus subtilis was reported as a potential antibiofilm against Streptococcus group A biofilm formation on seaweed. DTBP is the active principle compound for this antibiofilm resulting in changes in cell surface architecture with reducing thickness after treatment as reported by Viszwapriya et al. (2016). Bacillus subtillus filtrate proved to contain fatty acids i.e., palmitic and oleic acids which have role in plant protection against pathogens whether as antioxidant or antimicrobial as 
mentioned by many workers (Agoramoorthy et al., 2007, Chandrasekharan et al., 2008; Liu et al. 2008 and Lima et al., 2011).

GC-MS analysis detected some other compounds in B. subtillus filtrate such as: butyl alcohol, glycerin, butyl citrate, acetic acid hydroxyl (glycolic acid). Glycerin is used as a natural antimicrobial in food and cosmetics technologies (Idan et al., 2017) in addition to its medical benefits (Stout and McKessor, 2012 and Mortensen et al., 2017). This was in accordance with Cazorla et al. (2007) and Elkahouil et al. (2014) who found that Bacillus sp secrete butanolic compounds in their extracts and lipopeptides belonging to the surfactin, iturin and fengycin families. For example, fengycin is an antifungal lipopeptide complex produced by B. subtillus F-29-3 and the compound iso- hexadecanoic acid (1-16) is one of its structures (Vanittanakon et al., 1986).

Streptomyces albidoflavus Emeranaa strain MK203832.1 filtrate proved to contain another fatty acid, stearic acid, which have role in plant protection against pathogens whether as antioxidants or as antimicrobials as Agoramoorthy et al. (2007) and Ali et al. (2017) found. Data are in accordance with Idan et al. (2017) who found that the presence of fatty acids i.e. stearic acid, oleic acid, palmitic acid in PDA medium treated with Aspergillus niger filtrate reduced the mycelium growth. In actinomyces filtrates, some other compounds were detected, such as, undecane, acetyl phenylalanine (a product of phenylalanine $\mathrm{N}$ - acetyltransferase in the pathway of phenylalanine metabolism) and vanillin which can be used as a precursor in morphine synthesis, which have a role in systemic resistance induction and have herbicidal and fungicidal activities (Carpinella et al., 2003; Deba et al., 2007 and Lee et al., 2012).

Detection of components of these efficient endophytes filtrates interprets their mode of action in the lab or greenhouse experiments. All compounds play direct or indirect role in plant protection. Some actions belong to induced systemic resistance (ISR) with phenolic compounds which have antioxidant abilities (Pan et al., 2017).

Others depend on chemical reactions created by butanolic compounds or fatty acids which are efficient as antibiotics. Some of these detected compounds act as biosurfactants i.e., DTBP, butyl alcohol and butanone which are used as biodegradables. They are environmentally safe, stable under different conditions and can be produced from renewable inexpensive substrates when they are compared with synthetic compounds (Benincasa et al., 2010 and De Faria et al. 2011).

To date, no report is available on the isolation of endophytes from rice plants especially, S. albidoflavus and B. subtillus and their potentiality to control blast disease in Egypt. However, optimization of fermentation, media and extraction process is needed for maximum better results.

\section{A c know ledge ment}

The author thanks Dr. Amero. A. Emeran Professor of Plant Pathology, Faculty of Agriculture, Kafrelsheikh University, for cooperation, continuous support and providing facilities required to perform TLC analysis. 


\section{Ref e r e n e s}

Abubakar, M.N. and Majinda, R.R.T. 2016. GC-MS analysis and preliminary antimicrobial activity of Albizia adianthifolia Schumach and Pterocarpus angolensis DC. Medicines, 3:1-9.

Agoramoorthy, G.; Chandrasekaran, M.; Venkatesalu, V. and Hsu, M.J. 2007. Antibacterial and antifungal activities of fatty acid methyl esters of the blindyour-eye mangrove from India. Braz., J. Microbiol., 38(4): 739-742.

Ali, A.; Javaid, A. and Shoaib, A. 2017. GC-MS analysis and antifungal activity of methanolic root extract of Chenopodium album against Sclerotium rolfsii. Planta Daninha, 35.

Alina, S.O.; Constantinscu, F. and Petruta, C.C. 2015. Biodiversity of Bacillus subtilis group and beneficial traits of Bacillus species useful in plant protection, Romanian Biotechnological Letters, 20(5): 10737-10750.

Anonymous, 1996. "Standard Evaluation System for Rice". $4^{\text {th }}$ edition, IRRI, Manila, Philippines. $52 \mathrm{p}$.

Banaras, S.; Javaid, A.; Shoaib, A. and Ahmed, E. 2017. Antifungal activity of Cirsium arvense extracts against Phytopathogenic fungus Macrophomina Phaseolina. Planta Daninha, 35:1-10.

Bauer, A.W.; Kirby, W.M.M.; Scherris, J.C. and Turck, M. 1966. Antibiotic susceptibility testing by a standardized single disk method. Am.J.Clin.Pathol., 45:493-496.

Benincasa, M.; Marques, A.; Pinazo, A. and Manresa, A. 2010. Rhamnolipid surfactants: alternative substrates, new strategies. In R. Sen (Ed.), Advances in experimental medicine and biology. USA:Springer, pp.170-184.

Bizzari, S.N.; Blagoev, M. and Kishi A. 2009. CEH marketing research reports: Plasticizers [internet]. Menlo Park (CA): SRI Consulting (SRIC). Available from: http://www.sriconsulting.com/CEH/Private/Reports/576.0000/.

Borriss R. 2011. 'Use of plant-associated Bacillus strains as biofertilizers and biocontrol agents.' In: Bacteria in Agrobiology. Maheshwari, D. (Ed.). Plant Growth Responses. Heidelberg: Springer; p. 41-76.

Brader, G.; Compant, S.; Mitter, B.; Trognitz, F. and Sessitsch, A., 2014. Metabolic potential of endophytic bacteria. Curr. Opin. Biotechnol. 27: 30-37.

Bressan, W. 2003. Biological control of maize seed pathogenic fungi by use of actinomycetes. Biocontrol, 48:233-240.

Burgess, D.R.; Bretag, T. and Keane, P.J. 1997. Biocontrol of seed borne Botrytis cinerea in chickpea with Gliocladium roseum. Plant pathology. 46:298-305.

Carpinella, M.C.; Giorda, L.M.; Ferrayoli, C.G. and Palacios, S.M. 2003. Antifungal effects of different organic extracts from Melia azedarach L. on 
phytopathogenic fungi and their isolated active components. J. Agric. Food Chem., 51: 2506-2511.

Cazorla F.M.; Romero D.; Pérez-García A.; Lugtenberg B.J.; Vicente A.D. and Bloemberg, G. 2007. Isolation and characterization of antagonistic Bacillus subtilis strains from the avocado rhizoplane displaying biocontrol activity, $J$. Appl. Microbiol.,103 (5):1950-9.

Chandrasekharan, M.; Kannathasan, K. and Venkatesalu, V. 2008. Antimicrobial activity of fatty acid methyl esters of some members of chenopodiacea. Z Natur forseh.C:63, 331-336.

Choudhary D.K.; Johri B.N. 2009. Interactions of Bacillus spp. and plants - with special reference to induced systemic resistance (ISR). Microbiological Research, 68: 1754-1759.

Chowdhury S.P.; Dietel, K. Rändler, M.; Schmid, M.; Junge, H.; Borriss, R.; Hartmann, A.; Grosch, R. 2013. Effects of bacillus amyloliquefaciens fzb42 on lettuce growth and health under pathogen pressure and its impact on the rhizosphere bacterial community. PLoS ONE, 8 (7): e68818.

Cook, A.E. and Meyers, P.R. 2003. Rapid identification of filamentous actinomycetes to the genus level using genus specific 16S rRNA gene restriction fragment patterns. Int. J. Systematic and Evolutionary Microbiol., 53:1907-1915.

Costa, L.E.O.; Queiroz, M.V.; Borges, A.C.; Moraes, C.A. and Araújo, E.F.2012. Isolation and characterization of endophytic bacteria isolated from the leaves of the common bean (Phaseolus vulgaris). Braz. J. Microbiol., 43(4):1562-1575.

Crawford, D.L.; Lynch, J.M.; Whipps, J.M. and Ousley, M.A. 1993. Isolation and characterization of actinomycetes antagonists of a fungal root pathogen. Appl. Environ. Microbiol. 59(11): 3899-3905.

CSR. Chemical Safety Report: Decamethylcyclopentasiloxane, CAS No. 541-02-6; 2010.

Deba, F.; Xuan, T.D.; Yasuda, M. and Tawata, S. 2007. Herbicidal and fungicidal activities and identification of potential phytotoxins from Bidens pilosa L. var. radiata Scherff. Weed Biology and Management: 7, 77-83.

De Faria, A.F.; Teodoro-Martinez, D. S.; de Oliveira Barbosa, G.N.; Vaz, B.G.; Silva, Í. S.; Garcia, J. S. 2011. Production and structural characterization of surfactin (C14/Leu7) produced by Bacillus subtilis isolate LSFM-05 grown on raw glycerol from the biodiesel industry. Process Biochem. 46, 1951-1957.

Dharni, S.; Gupta, S.; Maurya, A.; Samad, A.; Srivastava, S.K.; Sharma, A. and Patra, D.D. 2014. Purification, characterization and in vitro activity of 2,4-Ditert-butylphenol from Pseudomonas monteilii PsF84: conformational and molecular docking studies. J. Agric. Food Chem. 2, 62(26):6138-46. 
Duncan, B.D. 1955. Multiple range and Multiple F-test. Biometrics, 11:1-42.

Elkahoui1, S.; Djébali, N.; Mrabet, M.; Karkouch, I.; Hadj Ibrahim, A.; Kalai, L.; Pachkwel, S.; Tabbene, O. and Limam, F. 2014. Mass spectrometry identification of antifungal lipopeptides from Bacillus sp. strain BCLRB2 against Rhizoctonia solani and Sclerotinia sclerotiorum. Applied Biochemistry and Microbiology, 50(2): 161-165.

Fernandez, M.J.; Ferrando L.; Fernandez A.S. 2006. Molecular and functional diversity of endophytic bacteria from leaves of three rice varieties. In: eleventh international symposium on microbial ecology (ISME-11), Vienna, Austria, August 20-25.

Gangwar, M.; Dogra, S. and Sharma, N. 2011. Antagonistic bioactivity of endophytic actinomycetes isolated from medicinal plants.J.Adv.Lab.Res.Biol.,11(10):154158.

Gottschalck T. E. and McEwen G. N., Jr. eds. 2004. International Cosmetic Ingredient Dictionary and Handbook. 10th ed. Volume 1. Washington DC: The Cosmetic, Toiletry and Fragrance Association.

Gupta, C.D.; Dubey, R. C.; Kang, S.C. and Maheshwari, D.K. 2001. Antibiotic Mediated Necrotrophic Effect of Pseudomonas GRC2 against Two Fungal Plant Pathogens. Curr. Sci., 81: 91-94.

Harish, S.; Kavino, M.; Kumar, N.; Saravanakumar, D.; Soorianathasundaram, K. and Samiyappan, R. 2008. Biohardening with Plant Growth Promoting Rhizosphere and Endophytic bacteria induces systemic resistance against Banana bunchy top virus, Applied Soil Ecology, 39(2):187-200.

Hassan, S.W.M. 2016. Antibacterial, Anticoagulant and Anti-inflammatory Activities of Marine Bacillus cereus S1. J. Pure and Appl. Microbiol., 10(4): 2593-2606.

Hong, K.; Gao, A.; Xie, Q.; Gao, H.; zhuang, L.; Yu, H. and Mand Ruan, J. 2009. Actinomycetes for marine drug discovery isolation from Mangrove soils and plants in china microbiological research. Mar Drugs.,7(1), 24-44.

Idan, A.A.; Sijam, K.; Kadir, J.; Rashid, T.S.; Awla, H.K. and Alsultan, W. 2017. Biological control of Pyricularia oryzae using antifungal compounds produced by Aspergillus niger. American Journal of Plant Sciences, 8(10): 2445-2460.

Kaaria, P.; Matiru, V. and Ndungu, M.v. 2012. Antimicrobial activities of secondary metabolites produced by endophytic bacteria from selected indigenous Kenyan plants. African Journal of Microbiology Research. 6(45): 7253-7258.

Kadoma, Y.; Ito, S.; Atsumi, T. and Fujisawa, S. 2009. Mechanisms of cytotoxicity of 2- or 2,6-di-tert-butylphenols and 2- methoxyphenols in terms of inhibition rate constant and a theoretical parameter. Chemosphere, 74: 626-632. 
Kafur, A. and Khan, A.B. 2011. Isolation of endophytic actinomycetes from Catharanthes roseus (L.) G. Don leaves and their antimicrobial activity. Iranian, J. Biotechnology, 9(4): 302-306.

Kim, Y.B.; Choi, E.H.; Keum, G.; Kang, S.B.; Lee, D.H.; Koh, H.Y. and Kim, Y. 2001. An Efficient Synthesis of Morpholin-2-one Derivatives Using Glycolaldehyde Dimer by the Ugi Multicomponent Reaction, Organic Lett., 3 (26):4149- 4152.

Kumar P., Dubey R.C., Maheshwari D.K. 2012. Bacillus strains isolated from rhizosphere showed plant growth promoting and antagonistic activity against phytopathogens. Microbiol. Res., 167(8): 493-499.

Kumar S.; Stecher G.; Li M.; Knyaz C. and Tamura K. 2018. MEGA X: Molecular Evolutionary Genetics Analysis across computing platforms. Molecular Biology and Evolution, 35:1547-1549.

Kuster, E. and Williams. S.T. 1964. Selection of Media for Isolation of Streptomycetes. Nature, 202: 928-929.

Lee, B.; Farag, M.A.; Park, H.B; Kloepper, J.W; Lee, S.H. and Ryu, C.M. 2012 Induced Resistance by a Long-Chain Bacterial Volatile: Elicitation of Plant Systemic Defense by a C13 Volatile Produced by Paenibacillus polymyxa, PLoS ONE 7(11): e48744.

Lima, L.A.., Johann, S.; Cisalpino, P.S.; Pimenta, L.P. and Boaventura, M.A. 2011. In vitro antifungal activity of fatty acid methyl esters of the seeds of Annona cornifolia A.St.-Hil. (Annonaceae) against pathogenic fungus Paracoccidioides brasiliensis. Rev. Soc. Bras Med Trop, 44(6):777-780.

Liu, S.; Ruan, W.; Li, J.; Xu, H.; Wang, J.; Gao, Y. and Wang, J. 2008. Biological control of phytopathogenic fungi by fatty acids, Mycopathologia. 166(2):93-102.

Mannaa, M. and Kim, K.D. 2018. Biocontrol activity of volatile-producing Bacillus megaterium and Pseudomonas protegens against Aspergillus and Penicillium spp. Predominant in Stored Rice Grains: Study II. Mycobiology, 46(1): 52-63.

Mohanraj, G. and Sekar, T. 2013. Isolation and screening of actinomycetes from marine sediments for their potential to produce antimicrobials. Int. J. LifeSc. Bt \& Pharm. Res, 2(3), 115-126.

Mortensen, A.; Aguilar, F.; Crebelli, R. 2017. Re-evaluation of glycerol (E 422) as a food additive. EFSA J., 15(3).

Moustafa, M.F.M.; Alamri, S.A.; Taha, T.H. and Alrumman, S.A. 2013. In vitro antifungal activity of Argemone ochroleuca Sweet latex against some pathogenic fungi. African Journal of Biotechnology, 12(10):1132-1137.

Naik, B.S.; Shashikala, J. and Krishnamurthy, Y.L. 2009. Study on the diversity of endophytic communities from rice (Oryza sativa L.) and their antagonistic activities in vitro. Microbiological research, 164(3): 290-296. 
Noell A.C.; Ely T.; Bolser D.K.; Darrach H.; Hodyss R.; Johnson P.V.; Hein J.D. and Ponce A. 2015. Spectroscopy and viability of Bacillus subtilis spores after ultraviolet irradiation: Implications for the detection of potential bacterial life on Europa. Astrobiology. 15(1): 20-31.

Okazaki, T. 2003. Studies on actinomycetes isolated from plant leaves. In: selective isolation of rare actinomycetes. kurtboke D.I. (Ed.). Queensland Complete Printing Services. Australia, pp 102-121.

Ou, S.H. 1985. Rice Diseases. $2^{\text {nd }}$ ed. Common wealth Mycological Institute, Kew, England, $380 \mathrm{p}$.

Pan, F.; Su, T.J.; Cai, S.M. and Wu, W. 2017. Fungal endophyte-derived Fritillaria unibracteata var. wabuensis: diversity, antioxidant capacities in vitro and relations to phenolic, flavonoid or saponin compounds, Sci Rep. 6:7.

Piroeva, I.; Vladimirova, S.; Dimowa, L.; Sbirkova, H.; L. 2013. A simple and rapid scanning electron microscope preparative technique for observation of biological samples: Application on bacteria and DNA samples. Bulgarian Chemical Communications,45(4):510-515.

Płaza, G.; Chojniak, J.; Rudnicka, K.; Paraszkiewicz, K. and Bernat, P. 2015. Detection of biosurfactants in Bacillus species: genes and products identification. J. Appl. Microbiol., 119: 1023-1034.

PMRA, Pest Management Regulatory Agency. 2005. Regulatory Note REG2005-01, PMRA List of Formulants [Internet]. Ottawa (ON): Pest Management Regulatory Agency, Health Canada. [cited 2008 January].

Pohl, C.H.; Kock, J.L.F. and Thibane,V.S. 2011. Antifungal free fatty acids: A Review, Science against microbial pathogens: communicating current research and technological advances, A. Méndez-Vilas (Ed). Formatex: 61-71.

Porter, J.N.; Wilhem, J.J. and Tresner, H.D. (1960). Method for the preferential isolation of actinomycetes from soils. Appl.Microbiol., 8(3): 174-178.

Qin L. and Judith L.K. 1999. Preparative thin layer chromatographic separation and subsequent gas chromatographic -mass spectrometric analysis of monoacylglycerols derived from butter oil by fungal degradation. $J$. Chromatogr A. 855(2):617-24.

Rajer, F.U.; Wu, H.; Xie, Y.; Xie, S.; Raza, W.; Tahir, H.A. and Gao, X. 2017. Volatile organic compounds produced by a soil-isolate, Bacillus subtilis FA26 induce adverse ultra-structural changes to the cells of Clavibacter michiganensis ssp. sepedonicus, the causal agent of bacterial ring rot of potato. Microbiology, 163(4):523-30.

Saitou N. and Nei M. 1987. The neighbor-joining method: A new method for reconstructing phylogenetic trees. Molecular Biology and Evolution, 4(4):406-25. 
Saleh, M. Mona 2012. Studies On The Sheath Rot Disease Of Rice. Ph.D. Thesis, Faculty of Agric., Kafr El-Sheikh Univ. Egypt, 147 p.

Sang, M.K.; Kim, J.D.; Kim, B.S. and Kim, K.D. 2011. Root treatment with rhizobacteria antagonistic to Phytophthora blight affects anthracnose occurrence, ripening and yield of pepper fruit in the plastic house and field. Phytopathology, 101: 666-678.

Sang, M.K. and Kim, K.D. 2012. The volatile- producing Flavobacterium johnsoniae strain GSE09 shows biocontrool activity against Phytophthora capsici in pepper. J.Appl. microbiol.113(2):383-398.

Santoyo, G.; Moreno-Hagelsieb, G.; del Carmen Orozco-Mosqueda, M. and Glick, B. R. 2016. Plant growth-promoting bacterial endophytes, Microbiol Res., (2):183:92-9.

Sella S.R.B.R.; Vandenberghe L.P.S. and Soccol C.R. (2014). Bacillus atrophaeus: main characteristics and biotechnological applications - a review. Crit Rev Biotechnol, 35(4): 1-13.

Shrilling, E.B. and Gottlieb, D. 1966. Methods of characterization of Streptomyces species. Int.J.Sys.Bacteriol.16:313-340.

Sivanandhan, S; Ganesan, P.; Jackson, A.; Darvin, S.; Paulraj, M.G. and Ignacimuthu, S. 2018. Activity of Some Medicinal Plants Against Phytopathogenic Fungi. International Journal of Scientific Research in Biological Sciences, 5(5):124137.

Stout, E.I. and McKessor. A. 2012. Glycerin-Based Hydrogel for Infection Control. Adv. wound care, 1(1):48-51.

Sukanya, S.L.; Yamini, D. and Fathima, S.K. 2011. Eco-friendly management of Pyricularia oryzae -The causal agent of blast of paddy. Current Botany, 2(8): 46-49.

Suryadi, Y.; Susilowati, D. N.; Putri, K. E. and Mubarik, N.R. 2011. Antagonistic activity of indigenous Indonesian bacteria as the suppressing agent of rice fungal pathogen. J.Internat,1Environ Appl Sci 6(4):558-568.

Taechowisan, T.; Lu, C.; Shen, V. and Lumyong, S. 2005. Secondary metabolites from endophytic Streptomyces aureofaciens CMUAc 130 and their antifungal activity. Microbiology, 151: 1691-5.

Taechowisan, T.; Peberdy, J.F. and Lumyong, S. 2003. Isolation of endophytic actinomycetes from selected plants and their antifungal activity. World j.Microbiol and Biotechnol, 19:381-5.

Takeshita, A.; Igarashi-Migitaka, J.; Nishiyama, K.; Takahashi, H.; Takeuchi, Y.; and Koibuchi, N. 2011. Acetyl tributyl citrate, the most widely used phthalate substitute plasticizer, induces cytochrome p450 3a through steroid and xenobiotic receptor. Toxicol Sci. 123(2):460-70. 
Tamura K.; Nei, M. and Kumar S. 2004. Prospects for inferring very large phylogenies by using the neighbor-joining method.Proc. Nati Acad. Sci. USA, 101(30):11030-5.

Tian, X.L.; Cao, L.X.; Tan, H.M.; Zeng, Q.G.; Jia, Y.Y. and Han, W.Q. 2004. Study on the communities of endophytic fungi and endophytic actinomycetes from rice and their antipathogenic activities in vitro. World J. Microbial Bitechnol, 20: $303-9$.

Vanittanakom, N.; Loeffler, W.; Koch, U. and Jung, G. 1986. Fengycin - A novel antifungal lipopeptide antibiotic produced by Bacillus subtillus F-29-3. J. Antibiotics, 39(7): 888- 901.

Varsha, K.K.; Devendra, L.; Shilpa, G.; Priya, S.; Pandey, A. and Nampoothiri, K.M. 2015.2,4-Di-tert-butylphenol as the antifungal, antioxidant bioactive purified from a newly isolated Lactococcus sp. Int. J. food microbiol.15(211):44-50.

Viszwapriya, D.; Prithika, U.; Deebika, S.; Balamurugan, K. and Pandian, SK.(2016). In vitro and in in vivo antibiofilm potential of 2,4-Di-tert-butylphenol from seaweed surface associated bacterium Bacillius subtilis against group A Streptococcus. Microbiol. Res. 191(10): 19-31.

Wang, H. C., Li, W. H., Chen, Q. Y., Huang, Y. F., Li, K., Xia, H. Q., Wang, M. S., Cai, L. T., Shang, S.H., and Shi, J. X. 2012. A rapid microbioassay for discovery of antagonistic bacteria for Phytophthora Parasitica var. nicotianae, Phytopathology 102:267-271.

Wu, W-J. ; Park, S-M. and Ahn, B-Y. 2013. Isolation and Characterization of an Antimicrobial Substance from Bacillus subtilis BY08 Antagonistic to Bacillus cereus and Listeria monocytogenes, Food Sci. Biotechnol. 22(2): 433-440.

Zhang, J. 2011. Improvement of an isolation medium for actinomycetes. Modern Applied Science. 5(2): 124-127.

Zhang, M.; Li, J.; Shen, A.; Tan, S.; Yan, Z.; Yu, Y. ; Xue, Z.; Tan, T. and Zeng, L. 2016. Isolation and Identification of Bacillus amyloliquefaciens IBFCBF-1 with Potential for biological control of Phytophthora blight and growth promotion of pepper. J. Phytopathol., 164(11-12):1012-1021.

Received 10/04/2018;

in revised form 10/05/2018) 


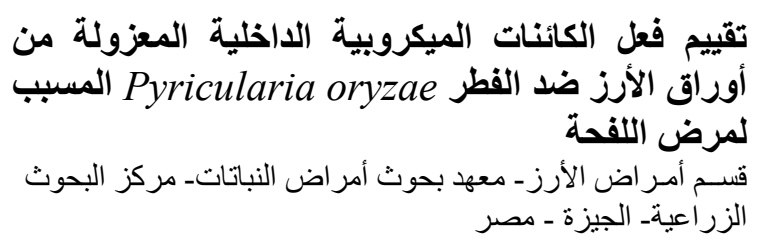

يُعد مرض لفحة الأرز المتسبب عن بيركيو لاريا أوريزا (ماجنابورس

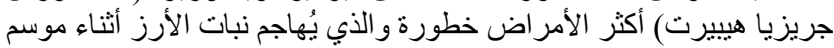

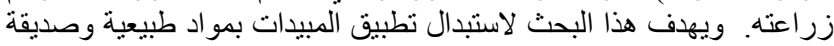

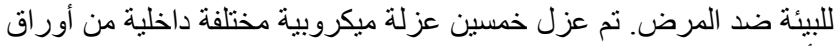

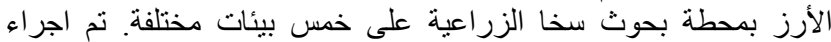

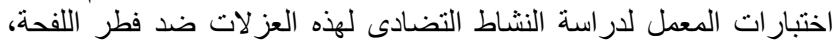

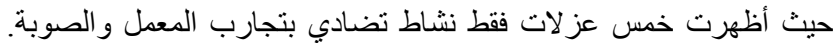

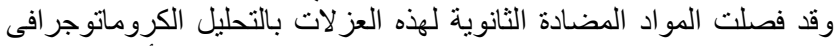

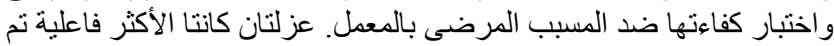

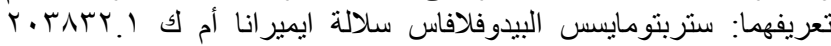

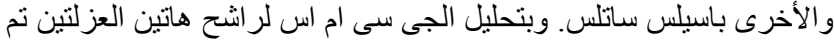
تعريف ع ب و با مركب على التو الي لهذه العزلات. 